RESEARCH

\title{
PIK3CA mutations in ductal carcinoma in situ and adjacent invasive breast cancer
}

\author{
Marie Colombe Agahozo', Anieta M Sieuwerts², S Charlane Doebar1, Esther I Verhoef1, Corine M Beaufort², \\ Kirsten Ruigrok-Ritstier ${ }^{2}$, Vanja de Weerd ${ }^{2}$, Hein F B M Sleddens ${ }^{1}$, Winand N M Dinjens ${ }^{1}$, John W M Martens ${ }^{2}$ and \\ Carolien H M van Deurzen'1
}

1Department of Pathology, Erasmus MC Cancer Institute, Rotterdam, The Netherlands

2Department of Medical Oncology, Erasmus MC Cancer Institute, Rotterdam, The Netherlands

Correspondence should be addressed to M C Agahozo: m.agahozo@erasmusmc.nl

\begin{abstract}
PIK3CA is one of the most frequently mutated genes in invasive breast cancer (IBC). These mutations are generally associated with hyper-activation of the phosphatidylinositol 3-kinase signaling pathway, which involves increased phosphorylation of AKT (p-AKT). This pathway is negatively regulated by the tumor suppressor PTEN. Data are limited regarding the variant allele frequency (VAF) of PIK3CA, PTEN and p-AKT expression during various stages of breast carcinogenesis. Therefore, the aim of this study was to gain insight into PIK3CA VAF and associated PTEN and p-AKT expression during the progression from ductal carcinoma in situ (DCIS) to IBC. We isolated DNA from DCIS tissue, synchronous IBC and metastasis when present. These samples were prescreened for PIK3CA hotspot mutations using the SNaPshot assay and, if positive, validated and quantified by digital PCR. PTEN and p-AKT expression was evaluated by immunohistochemistry using the Histo-score (H-score). Differences in PIK3CA VAF, PTEN and p-AKT H-scores between DCIS and IBC were analyzed. PIK3CA mutations were detected in 17 out of 73 DCIS samples, 16 out of 73 IBC samples and 3 out of 23 lymph node metastasis. We detected a significantly higher VAF of PIK3CA in the DCIS component compared to the adjacent IBC component $(P=0.007)$. The expression of PTEN was significantly higher in DCIS compared to the IBC component in cases with a wild-type (WT) PIK3CA status $(P=0.007)$, while it remained similar in both components when PIK3CA was mutated. There was no difference in $\mathrm{p}$-AKT expression between DCIS and the IBC component. In conclusion, our data suggest that PIK3CA mutations could be essential specifically in early stages of breast carcinogenesis. In addition, these mutations do not co-occur with PTEN expression during DCIS progression to IBC in the majority of patients. These results may contribute to further unraveling the process of breast carcinogenesis, and this could aid in the development of patient-specific treatment.
\end{abstract}

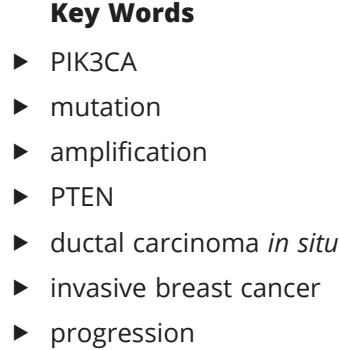

Endocrine-Related Cancer (2019) 26, 471-482

\section{Introduction}

Invasive breast cancer (IBC) is a heterogeneous disease, consisting of several molecular subtypes, all with a specific tumor biology and clinical outcome (Perou et al. 2000). Each of these molecular subtypes, which are based on microarray-based gene expression studies, also have an immunohistochemical surrogate subtype based 
on estrogen receptor (ER), progesterone receptor (PR), human epidermal growth factor receptor (HER2) and Ki-67 (Goldhirsch et al. 2013). The somatic mutation spectrum varies widely across these different subtypes (Perou et al. 2000). One of the most frequently described mutations in IBC are present in phosphatidylinositol-4,5biphospate-3-kinase, catalytic subunit alpha (PIK3CA) and occur in 16-45\% of all patients (Cancer Genome Atlas Network 2012, Ang et al. 2014, Pang et al. 2014, Abba et al. 2015, Mukohara 2015). The majority of these cases are E542K and E545K mutations, located in exon 9, and H1047R mutations, located in exon 20 of the gene (Vogt et al. 2007). These mutations are mainly observed in ER+ IBC subtypes, which generally have a good prognosis (Kalinsky et al. 2009, Cizkova et al. 2012, Pang et al. 2014, Zardavas et al. 2018). PIK3CA mutations are associated with hyper-activation of the phosphatidylinositol 3-kinase (PI3K) signaling pathway, which involves increased phosphorylation of AKT (p-AKT) and results in increased cell growth and survival (Vivanco \& Sawyers 2002, Chalhoub \& Baker 2009). This pathway is negatively regulated by phosphatase and tensin homologue deleted on chromosome 10 (PTEN) (Chalhoub \& Baker 2009). Therefore, impaired PTEN function or loss of PTEN expression also results in uncontrolled activation of the PI3K signaling pathway. PTEN loss is generally associated with a worse disease-free and overall survival (Bose et al. 2002, Lee et al. 2004, Iqbal et al. 2012, Li et al. 2017). On the other hand, PIK3CA mutations are generally associated with longer recurrence-free survival in IBC patients, though associated with reduced therapy response in HER2+ IBC subtypes (Berns et al. 2007, Loi et al. 2010, Cizkova et al. 2012, Sueta et al. 2014). A reduced therapy response in HER2+ IBC subtypes was also shown in cases with an aberrant PTEN expression (Nagata et al. 2004, Gschwantler-Kaulich et al. 2017).

Approximately $50 \%$ of all IBCs are associated with a synchronous precursor lesion, of which ductal carcinoma in situ (DCIS) is the most common (Barnes et al. 2012, Bleyer \& Welch 2012). PIK3CA mutations already arise in these pre-malignant lesions. They are reported in $30 \%$ of patients with pure DCIS (without an invasive component) and in $24-43 \%$ of all DCIS cases with an adjacent invasive component (Li et al. 2010, Miron et al. 2010, Troxell 2012, Ang et al. 2014). These reported PIK3CA mutation frequencies in DCIS are similar to those reported in IBC (Li et al. 2010, Miron et al. 2010). Furthermore, the type of PIK3CA mutation remains the same throughout breast cancer progression. Next to these concordances, several studies also reported a discordant PIK3CA mutation status in synchronous DCIS and IBC (Miron et al. 2010, Dupont Jensen et al. 2011, Hernandez et al. 2012). However, data are limited with respect to the variant percentage of PIK3CA mutations during progression from DCIS to IBC.

Loss of PTEN expression has been described in $11-44 \%$ of cases with pure DCIS, in $35 \%$ of cases with synchronous DCIS and IBC and in 12-38\% of cases with IBC (Bose et al. 2002, Song et al. 2010, Knudsen et al. 2012, Sakr et al. 2014, Wang et al. 2017). In synchronous DCIS and IBC, PTEN expression was similar in the DCIS and the invasive component (Sakr et al. 2014). Studies correlating PIK3CA and PTEN mutations suggest mutual exclusivity (Saal et al. 2005, cBioPortal 2015), although data regarding PTEN expression during breast cancer progression are limited.

Current literature regarding p-AKT expression is mainly focused on IBC. Expression of p-AKT has been reported in up to $75 \%$ of IBC cases (Esteva et al. 2010, Sakr et al. 2014, Sueta et al. 2014, Cossu-Rocca et al. 2015, Azim et al. 2016, Guarneri et al. 2019). PIK3CA mutations are associated with high p-AKT expression in triple negative and metastatic breast cancer (Dupont Jensen et al. 2011, Cossu-Rocca et al. 2015, Azim et al. 2016). Expression of p-AKT has also been described in $42 \%$ of cases with pure DCIS and in $70 \%$ of cases with synchronous DCIS and IBC (Sakr et al. 2014). In matched DCIS and IBC, the expression was similar (Sakr et al. 2014). The effect of PIK3CA mutations on p-AKT expression at the DCIS stage has to be investigated.

Since PIK3CA mutations are early events in breast carcinogenesis, they might be critical in the earliest steps of breast cancer initiation. Furthermore, it is of interest to know how this might be associated with PTEN and p-AKT expression. Added knowledge on the variant allele frequency (VAF) of PIK3CA and its association with PTEN and p-AKT expression during breast cancer progression could facilitate future development of early intervention studies. Therefore, the aim of this study was to have a better understanding of breast carcinogenesis by in-depth analyses of the presence and VAF of PIK3CA mutations and their relation to PTEN and p-AKT expression during the progression from DCIS to IBC.

\section{Materials and methods}

\section{Patient selection}

Patients diagnosed with synchronous DCIS and adjacent IBC and treated at the Erasmus Medical Center - Cancer Institute in the period between 2010 and 2015 were 
included $(n=73)$. Patients with neoadjuvant treatment, previous breast irradiation or a history of breast cancer were excluded. The tumor cell percentage was estimated beforehand by a pathologist and only samples with an estimated tumor cell percentage $\geq 50 \%$ were included. DCIS grade was assessed primarily based on cytonuclear differentiation and IBC grade was assessed according to the modified Bloom Richardson score (Elston \& Ellis 1991). Immunohistochemistry (IHC) was performed on each IBC, using ER (ER SP1; Ventana Medical Systems, Inc.), PR (PR 1E2; Ventana Medical Systems, Inc.) and HER2 (HER2 4B5; Ventana Medical Systems, Inc.) antibodies. ER and PR status were scored positive when $\geq 10 \%$ of the tumor cells were positive, according to the Dutch Breast Cancer Guideline (NABON 2017). HER2 status was scored according to the international guidelines (Wolff et al. 2014). For this study, coded leftover patient material was used in accordance with the Code of Conduct of the Federation of Medical Scientific Societies in The Netherlands (FEDERA 2011). Therefore, there was no need for an informed consent or study approval by an ethical committee. Figure 1 provides an overview of the workflow.

\section{DNA and RNA isolation, sample enrichment and product validation}

In addition to the estimated tumor cell percentage assessed by a pathologist, we analyzed EPCAM, ESR1 and $E R B B 2$ gene expression in all lesions. For this, $8 \mu \mathrm{m}$ thick formalin-fixed paraffin-imbedded (FFPE) tissues of DCIS and adjacent IBC and, if available, corresponding lymph node and distant metastasis were dewaxed and rehydrated, followed by a haematoxylin staining. Subsequently, tissue was microdissected manually, using a sterile scalpel under a stereomicroscope (Zeiss).

Microdissected cells were stored into RNAse/Dnasefree tubes containing RNALater (Thermo Fisher) at $-80^{\circ} \mathrm{C}$. RNALater was removed and replaced by xylene after which RNA and DNA isolation was performed with the AllPrep RNA/DNA FFPE isolation kit (Qiagen) according the manufacturer's instructions. RNA concentrations were measured by Nanodrop (Thermo Fisher Scientific) and DNA concentrations with picogreen by Qubit (Thermo Fisher Scientific) and stored at $-30^{\circ} \mathrm{C}$.

The isolated RNA was used to generate cDNA and analyze the ESR1, ERBB2 and EPCAM expression by reverse transcriptase RT-PCR as previously described (Doebar et al. 2017, Sieuwerts et al. 2017). In brief, a gene-specific preamplification was performed for ESR1 (Hs00174860_m1),
SNaPshot analysis on total number of patients $(n=73)$

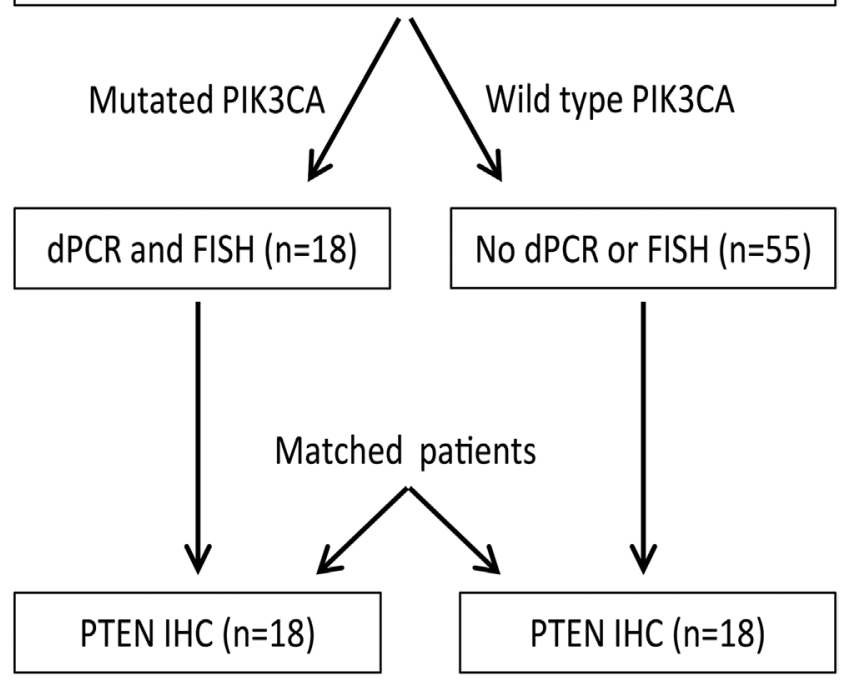

Figure 1

Flowchart of the analyzed samples. dPCR, digital PCR; FISH, fluorescent in situ hybridization; IHC, immunohistochemistry. Patients were matched on $E R, P R, H E R 2$ and tumor grade.

ERBB2 (Hs01001580_m1) and EPCAM (Hs00158980_m1) and three reference genes (GUSB, Hs9999908_m1, HMBS, Hs00609297_m1 and HPRT1) using the TaqMan PreAmp Master mix (Thermo Fisher Scientific) for 15 cycles, followed by TaqMan probe-based real-time PCRs according to the manufacturer's instructions in a MX3000P RealTime PCR System (Agilent). Gene expression levels were quantified relative to the average expression of GUSB, HMBS and HPRT1 using the $2 \wedge$ (average quantitative cycle (Cq) reference genes - Cq target gene) method.

Prior to use, DNA samples were diluted or (Sieuwerts et al. 2017) vacuum dried to a working concentration of $1 \mathrm{ng} / \mu \mathrm{L}$ in case the original concentration was $\leq 0.5 \mathrm{ng} / \mu \mathrm{L}$. Next, the PIK3CA targets were enriched in a targeted PCR using TaqMan PreAmp mastermix (Thermo Fisher Scientific) and PIK3CA-specific exon 9 and exon 20 region primers at a final concentration of $10 \mathrm{nM}$ in a Thermal cycler (Bio-Rad Laboratories, Inc.) (Supplementary Table 1, see section on supplementary data given at the end of this article). These pre-amplified products were then diluted $10 \times$ in LoTE ( 3 mM Tris-HCl/0.2 mM EDTA, pH 8.0) before further amplification by SYBR-based quantitative PCR (qPCR), using the same primers at a final concentration of $100 \mu \mathrm{M}$ and SensiFast low ROX mastermix (Bioline). The resulting qPCR products were used for fragment size validation and quantification of the fragment concentrations by microchip electrophoresis with the DNA-500 kit (MultiNA, Shimadzu) and SNaPshot analysis. (c) 2019 Society for Endocrinology Published by Bioscientifica Ltd. Printed in Great Britain 


\section{Detection of PIK3CA mutations by SNaPshot assay}

SNaPshot is a rapid and simple assay to detect multiple PIK3CA mutations present in down to $5-10 \%$ of the tumor cells in a single analysis (Boland et al. 2003, Fariña Sarasqueta et al. 2011). The SNaPshot Multiplex System for SNP Genotyping (Thermo Fisher Scientific) was therefore used to identify PIK3CA mutations in our cohort. The qPCR products were first diluted in LoTE to a working concentration of $2.5 \mathrm{ng} / \mu \mathrm{L}$ and were then amplified, using the PIK3CA primers at a final concentration of $1 \mu \mathrm{M}$ for exon 9 and $0.7 \mu \mathrm{M}$ for exon 20 (Supplementary Table 1). Then, samples were purified using $3 \mathrm{U} /$ Well FastAP and $0.45 \mathrm{U} /$ Well Exonuclease 1 (Thermo Fisher Scientific) after which the SNaPshot ddNTP multiplex probe mix (Supplementary Table 2) was used to extend and terminate the reaction as previously described (Hurst et al. 2009). Finally, an ABI PRISM 3100 Genetic Analyzer (Thermo Fisher Scientific) was used to detect the mutations by electrophoresis. Breast cancer cell lines with known PIK3CA mutation status were used as positive and negative controls. Mutation analysis was performed using GeneMarker V2.7.0 Software (SoftGenetics, LLC).

\section{Quantifying PIK3CA mutation by dPCR assay}

Digital PCR (dPCR) is a highly sensitive technique that can detect and, in addition to the SNaPshot assay, quantify mutations present in down to $0.1 \%$ of tumor cells (Beaver et al. 2013, Sueta et al. 2014). We therefore used this technique to validate the SNaPshot results and quantify the relative number of PIK3CA-mutated copies in both the DCIS and IBC component of those patients with a PIK3CA mutation identified by SNaPshot analysis. Additionally, we also performed a dPCR assay on samples with a wildtype (WT) PIK3CA mutation ( $n=33$ samples) outcome by $\mathrm{SNaPshot} \mathrm{to} \mathrm{ensure} \mathrm{we} \mathrm{did} \mathrm{not} \mathrm{overlook} \mathrm{mutations.}$ Mutation-specific TaqMan SNP Genotyping Assays (Thermo Fisher Scientific) were used for dPCR analysis. These assays consist of two TaqMan probes, one for the WT sequence and the other one specific for E542K, E545K, H1047R or H1047L PIK3CA mutation (Supplementary Table 3). Starting with $1 \mathrm{ng} / \mu \mathrm{L}$ DNA, the PIK3CA targets where first enriched using Taqman PreAmp mastermix (Thermo Fisher Scientific) and the mutation-specific TaqMan SNP Genotyping Assays in a Thermal cycler (Bio-Rad Laboratories, Inc.), followed by a qPCR with the same assays and SensiFast Universal Mastermix (Bioline) to determine the optimum loading concentration in de dPCR chip (Supplementary Table 4). The mutation-specific
TaqMan SNP Genotyping assay was then added to these pre-amplified DNA samples along with the Digital PCR V2 Master Mix (Thermo Fisher) (Supplementary Table 5). Next, the samples were loaded to a Digital PCR 20K chip and sealed using the QuantStudio 3D Digital PCR Chip Loader, according to the manufacturer's instructions. After loading, a dPCR reaction was performed in a Thermo Fisher ProFlex PCR system. For optimal signal detection, two chip reads were performed using the Thermo Fisher QuantStudio 3D Digital PCR Chip Reader. The samples were individually analyzed online using the QuantStudio 3D AnalysisSuite. PIK3CA mutations in DCIS, IBC and lymph node metastasis were quantified as VAF, that is defined as the number of PIK3CA-mutated copies divided by the number of PIK3CA WT copies plus the number of PIK3CA mutated copies. Samples were scored as PIK3CA mutant when the VAF was at least 5\%.

\section{Assessing PIK3CA amplification by fluorescent in situ hybridization (FISH)}

Because the relative number of PIK3CA mutations might be influenced by amplification or deletion of the PIK3CA gene, we performed FISH on all patients with a PIK3CA mutation $(n=18)$, using a PIK3CA-specific FISH (ZytoLight SPEC PIK3CA/CEN 3 Dual Color Probe; Catalog number: Z-2140-200, Zytovision). For this assay, consecutive slides were used from the same tissue blocks as previously used for microdissection. First, the $4 \mu \mathrm{m}$-thick FFPE tissues were dewaxed, dehydrated and cooked for $13 \mathrm{~min}$ in citrate buffer. Subsequently, tissue was treated with pepsin for $20 \mathrm{~min}$ and dehydrated, followed by probe hybridizing for $10 \mathrm{~min}$ at $75^{\circ} \mathrm{C}$ and overnight at $37^{\circ} \mathrm{C}$ in a Hybridizer (Dako Agilent). The slides were washed in a stringent wash buffer at $73^{\circ} \mathrm{C}$, rinsed in $2 \times$ SSC, dehydrated airdried, sealed with a cover glass and stored at $4^{\circ} \mathrm{C}$ until further use.

For visualization and analysis, the stained slides were scanned by a wide field fluorescent microscope (Zeiss, Jena). At least three areas of the hybridized region, with the highest tumor cell content were imaged at a $63 \times$ magnification using a Z-stack of three layers and a 3-by-3 tile. The images were analyzed using a Fiji (ImageJ 1.49s) cell counter plugin. The PIK3CA and CEN3 signals were counted in at least 100 cells containing both signals. PIK3CA amplification was defined as a PIK3CA/CEN3 ratio $\geq 2.0$ and/or when the average number of PIK3CA alleles per tumor cell nucleus was $\geq 4.0$ (Shi et al. 2012, Kim et al. 2016). (c) 2019 Society for Endocrinology Published by Bioscientifica Ltd. Printed in Great Britain 


\section{Quantifying PTEN and p-AKT expression by IHC}

In order to assess the potential negative regulation of the PI3K pathway during progression, we performed PTEN and p-AKT IHC on consecutive slides. We included all patients with a PIK3CA mutation $(n=18)$ and matched patients with a WT PIK3CA status $(n=18)$. Matching was based on ER, PR, HER2 expression and tumor grade. OptiView DAB IHC Detection Kit (v1.00.0117, Ventana Medical Systems, Inc.) was used to detect PTEN expression. For this assay, we used the same tissue blocks as previously used for microdissection. Initially, $4 \mu \mathrm{m}$-thick FFPE tissues of DCIS and adjacent IBC were incubated on a hot plate over the weekend for optimal sealing of the slides. The staining was then carried out by a BenchMark ULTRA instrument (Ventana Medical Systems, Inc.), using a customized protocol for the PTEN monoclonal antibody (D4.3 XP, 1:200, Cell Signaling Technology) and p-AKT monoclonal antibody (Ser473, D9E XP, 1:100, Cell Signaling Technology). The slides were incubated in citrate buffer at $97^{\circ} \mathrm{C}$ for antigen retrieval and then with the primary antibody, each for $32 \mathrm{~min}$. Skin and breast epithelial cells were used as positive controls for PTEN. IBC and a breast cancer cell line (MM468) were used as positive controls for p-AKT. DCIS and IBC components were scored separately, blinded for PIK3CA status. PTEN and p-AKT expression was reviewed by two observers and assessed semi-quantitatively using the Histo-score (H-score) (Sakr et al. 2010). The total $\mathrm{H}$-score varied from 0 to 300 and was calculated as the staining intensity (0-3) multiplied by the percentage of positive cells $(0-100)$.

\section{Statistical analysis}

SPSS Statistics 21 (IBM) was used for statistical analysis. After testing for normal distribution, we used nonparametric tests to analyze the differences in PIK3CA VAF, PTEN and p-AKT expression. The Wilcoxon signedranks test was used to analyze the differences between paired samples and the Mann-Whitney test was used to analyze the differences in PTEN expression between patients with mutated and WT PIK3CA. To correlate linear variables, the Spearman rank correlation test was used. A Chi-square test was used to analyze the associations between groups. $P$ values $<0.05$ were considered statistically significant.

\section{Results}

\section{Clinicopathological characteristics}

A total number of 73 patients with paired DCIS and adjacent IBC were included (Table 1). Median age at diagnosis was 56 years, ranging from 28 to 102 years. Approximately half of the patients (53.4\%) underwent breast-conserving surgery and the majority (94.5\%) of patients underwent lymph node staging. Local metastasis was found in half of the examined lymph nodes and six patients developed a distant metastasis. In total, 23 lymph nodes metastasis and 3 distant metastatic lesions were included for PIK3CA mutation analysis.

\section{Presence of PIK3CA mutations in DCIS and adjacent IBC by SNaPshot analysis}

Overall, a PIK3CA somatic hotspot mutation was detected in $24.7 \%$ (18 out of 73 ) of patients (Fig. 2). The detected mutations were mainly located in H1047R $(n=9)$, followed by E545K $(n=5)$ and E542K $(n=4)$. A PIK3CA mutation

Table 1 Patient and tumor characteristics.

\begin{tabular}{|c|c|}
\hline Patient and tumor characteristics $(n=73)$ & Frequency (\%) \\
\hline \multicolumn{2}{|l|}{ Age at diagnosis (in years) } \\
\hline Median (range) & $56.7(28-102)$ \\
\hline \multicolumn{2}{|l|}{ Diameter IBC (cm) } \\
\hline Median (range) & $2.10(0.60-11.0)$ \\
\hline \multicolumn{2}{|l|}{ IBC histological grade } \\
\hline Low & $2(2.7)$ \\
\hline Intermediate & $28(38.4)$ \\
\hline High & $43(58.9)$ \\
\hline \multicolumn{2}{|l|}{ Diameter DCIS (cm) } \\
\hline Median (range) & $3.00(0.10-15.0)$ \\
\hline \multicolumn{2}{|l|}{ DCIS histological grade } \\
\hline Low & $1(1.4)$ \\
\hline Intermediate & $23(31.5)$ \\
\hline High & $49(67.1)$ \\
\hline \multicolumn{2}{|l|}{ IBC IHC subtype } \\
\hline $\mathrm{ER}+\mathrm{HER} 2-$ & $34(46.6)$ \\
\hline ER+ HER2+ & $15(20.5)$ \\
\hline ER- HER2+ & $9(12.3)$ \\
\hline ER-, PR-, HER2- & $15(20.5)$ \\
\hline \multicolumn{2}{|l|}{ Lymph node status } \\
\hline No metastasis or isolated tumor cells & $41(58.9)$ \\
\hline Micro- or macrometastasis & $28(35.6)$ \\
\hline No LN procedure & $4(5.5)$ \\
\hline \multicolumn{2}{|l|}{ Distant metastasis } \\
\hline Yes & $6(5.5)$ \\
\hline No & $67(94.5)$ \\
\hline
\end{tabular}

(c) 2019 Society for Endocrinology Published by Bioscientifica Ltd. Printed in Great Britain 


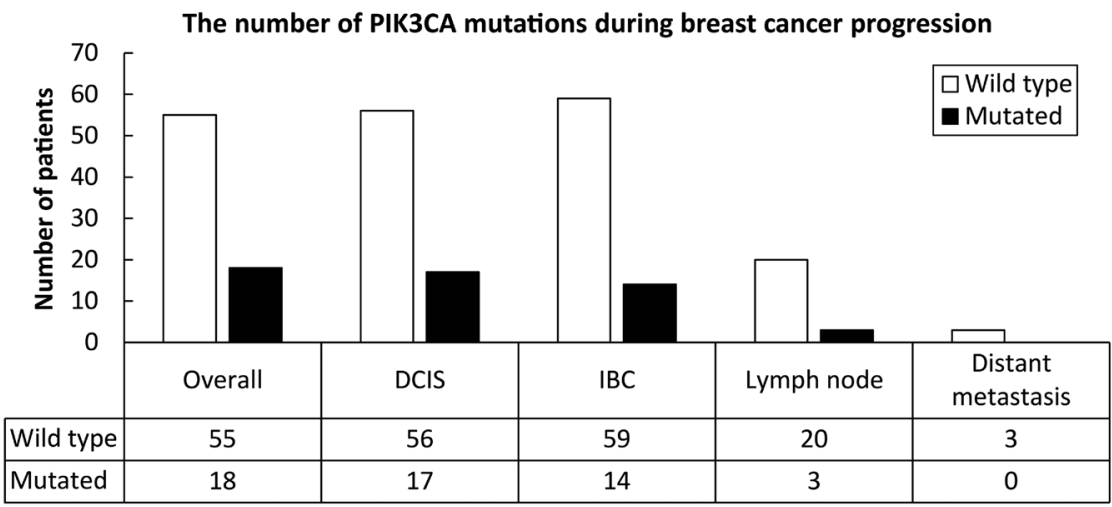

\section{Figure 2}

The number of PIK3CA mutations during breast cancer progression. Overall, 18 patients with a mutated PIK3CA status (black bars) and 55 patients with a WT PIK3CA status (white bar) were identified.

was identified in both the DCIS and the IBC component of 14 patients. A discordant PIK3CA mutation status was found in four patients, whereby three patients had a PIK3CA mutation in the DCIS component only and one patient had a PIK3CA mutation in the IBC component only. A PIK3CA mutation was detected in 3 out of the 23 patients with a lymph node metastasis. In these three patients, a PIK3CA mutation was also present in both the DCIS and IBC stage. In our cohort, none of the patients with a PIK3CA mutation in either the DCIS component and/or the invasive component developed a distant metastasis. Furthermore, no PIK3CA mutations were identified in the three available distant metastases of three patients without a PIK3CA mutation in the DCIS and IBC component. When a PIK3CA mutation was detected in the DCIS and/or IBC component of a patient, this concerned the same mutation in all cases. We never observed two or more different mutations in the material of one patient.

There were no significant associations between the presence of a PIK3CA mutation in either the DCIS or IBC component and clinicopathologic characteristics. However, the majority of samples with a PIK3CA mutation, 11 out of 18 patients, were ER+ and HER2-. This result was not affected by using a cut-off for ER/PR positivity of $1 \%$.

\section{VAF of PIK3CA in DCIS and adjacent IBC by dPCR analysis}

All PIK3CA hotspot mutations, as previously detected by SNaPshot analysis, were also detected by dPCR. However, dPCR detected one additional patient with a $6.15 \%$ PIK3CA VAF in an IBC lesion, which was not detected by the SNaPshot assay. In this patient, the SNaPshot assay only detected a mutation in the DCIS component. Other samples with a WT PIK3CA outcome by SNaPshot remained WT after sample check with dPCR. In summary, after validation by dPCR, the total number of discordant PIK3CA mutation status between DCIS and IBC was reduced from four to three patients: two patients had a PIK3CA mutation in the DCIS component only and one patient had a mutation in the invasive component only.

After quantification, we found a higher PIK3CA VAF in the DCIS stage $(45.8 \%)$ than in the IBC stage $(31.7 \%)$, $P$ value $=0.007$ (Fig. 3), which could not be caused by differences in tumor cell percentage as the mean tumor cell percentage in DCIS was similar to that in IBC $(P$ value $=0.24)$ and results were not affected by correction for tumor cell percentage $(P$ value $=0.018)$. Additionally, the expression of the epithelial marker EPCAM, ESR1 expression and ERBB2 expression were similar in DCIS and $\operatorname{IBC}(P=0.910, P=0.177$ and $P=0.381$ respectively).

All three patients with a PIK3CA mutation in the lymph node metastases also had a PIK3CA mutation in both the DCIS and the IBC component. The PIK3CA VAF in lymph node metastases were similar to that found in the IBC stage (33.8 vs $30.9 \%)$. In one patient with a PIK3CA mutation in the DCIS stage only, no PIK3CA mutation was detected in the lymph node metastasis (Supplementary Fig. 1).

\section{Assessing PIK3CA amplification by fluorescent in situ hybridization (FISH)}

Using FISH we detected PIK3CA amplification in 3 out of 18 patients (Fig. 4; patients 3, 4 and 8). The PIK3CA VAF by dPCR in these patients was 70.5, 51.1 and $77.0 \%$ respectively. The number of mutant PIK3CA DNA copies in patients 3 and 8 were up to three times higher than the number of WT PIK3CA DNA copies. In patient 4 , the number of WT and the mutated PIK3CA DNA copies were similar (data not shown). With regard to breast cancer progression, PIK3CA amplification was observed only in the DCIS component. No PIK3CA amplification was
C) 2019 Society for Endocrinology Published by Bioscientifica Ltd. Printed in Great Britain 


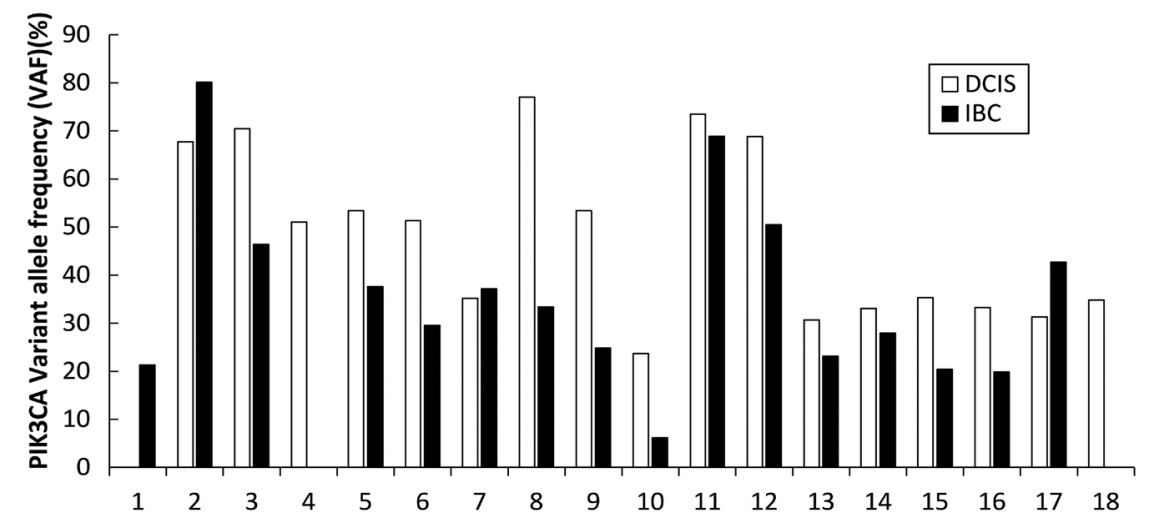

\begin{abstract}
Figure 3
The percentage PIK3CA VAF in paired DCIS (white bars) and IBC (black bars). In total, 2 patients (patient number 4 and 18) had a PIK3CA mutation in the DCIS component only and one patient (patient number 1) had a mutation in the invasive component only. The $X$-axis displays the number of analyzed patients and the $Y$-axis displays the PIK3CA VAF in \%. DCIS, ductal carcinoma in situ; IBC, invasive breast cancer.
\end{abstract}

observed in IBC lesions or lymph node metastasis. The differences in VAF remained significant after removal of PIK3CA-amplified cases.

\section{PTEN expression during breast cancer progression}

PTEN was expressed in the cytoplasm in the majority (29 out of 36) of examined patients. Nuclear PTEN expression was observed in two cases. Both patients had a WT PIK3CA status. Overall, PTEN expression had a significantly lower H-score in IBC compared to DCIS (mean \pm s.D. of $111.11 \pm 112.43$ vs $138.57 \pm 120.78$ respectively; $P$ value $=0.004$ ). Figure 5 illustrates a case with a higher PTEN expression in the DCIS component with a WT PIK3CA status as compared to the adjacent invasive component. When stratified by PIK3CA mutation status, PTEN expression was lower in the IBC component compared to the DCIS component in patients with a WT PIK3CA status (mean H-score \pm s.D. of $90.28 \pm 105.20$ vs $129.72 \pm 118.61$, respectively; $P$ value $=0.007$ ). In patients with a PIK3CA mutation on the other hand, there was no significant difference between PTEN expression in the DCIS component and the IBC component $(P$-value $=0.14)$.

\section{p-AKT expression during breast cancer progression}

P-AKT expression was evaluated on 36 patients (two DCIS samples were excluded due to tissue exhaustion). In total, there was cytoplasmic p-AKT expression in 15 out of 36 patients. Overall, p-AKT expression was low with a median H-score of 0 (range: 0-205 in DCIS and 0-45 in IBC). Seven out of the 15 patients with p-AKT expression had a PIK3CA mutation; the remaining eight cases were WT. Six out of 15 patients had p-AKT expression in DCIS and adjacent IBC. There was no difference in the level of p-AKT expression between these two components. The remaining nine cases showed a discordant p-AKT expression; four patients had expression in the DCIS component only and five patients had expression in de IBC component only. Figure 6 provides a heat map of these analyses.

\section{Discussion}

PIK3CA mutations have been frequently described in IBC. However, data are limited regarding its presence and quantity during progression from DCIS to IBC.
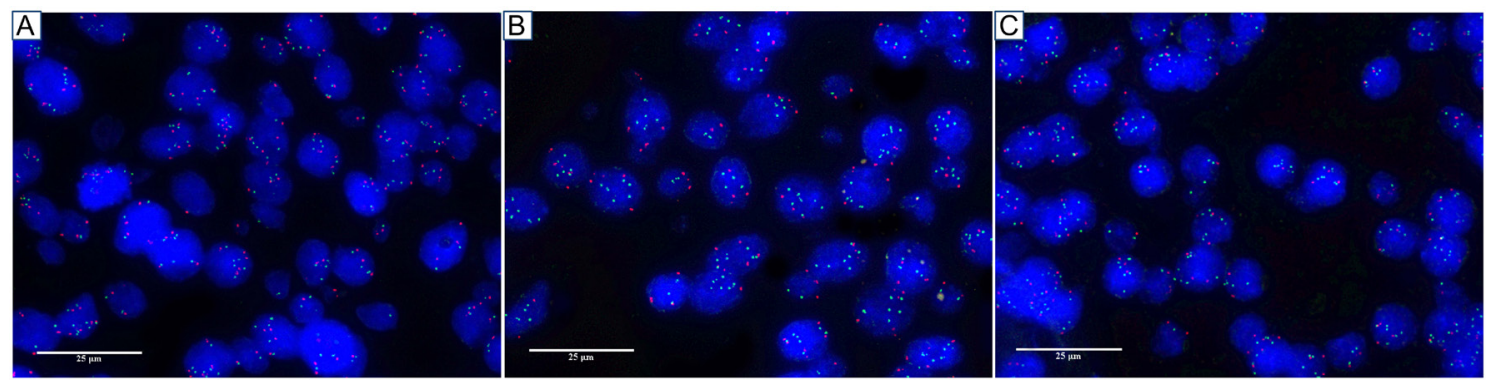

Figure 4

Representative FISH images (63× magnification) of cases with a PIK3CA amplification (number of PIK3CA/nucleus $\geq 4$ ) in DCIS of patient 3 (A), patient 4 (B) and patient 8 (C). Blue represents the cell nuclei, red dots represent CEN3 and the green dots represent PIK3CA. 

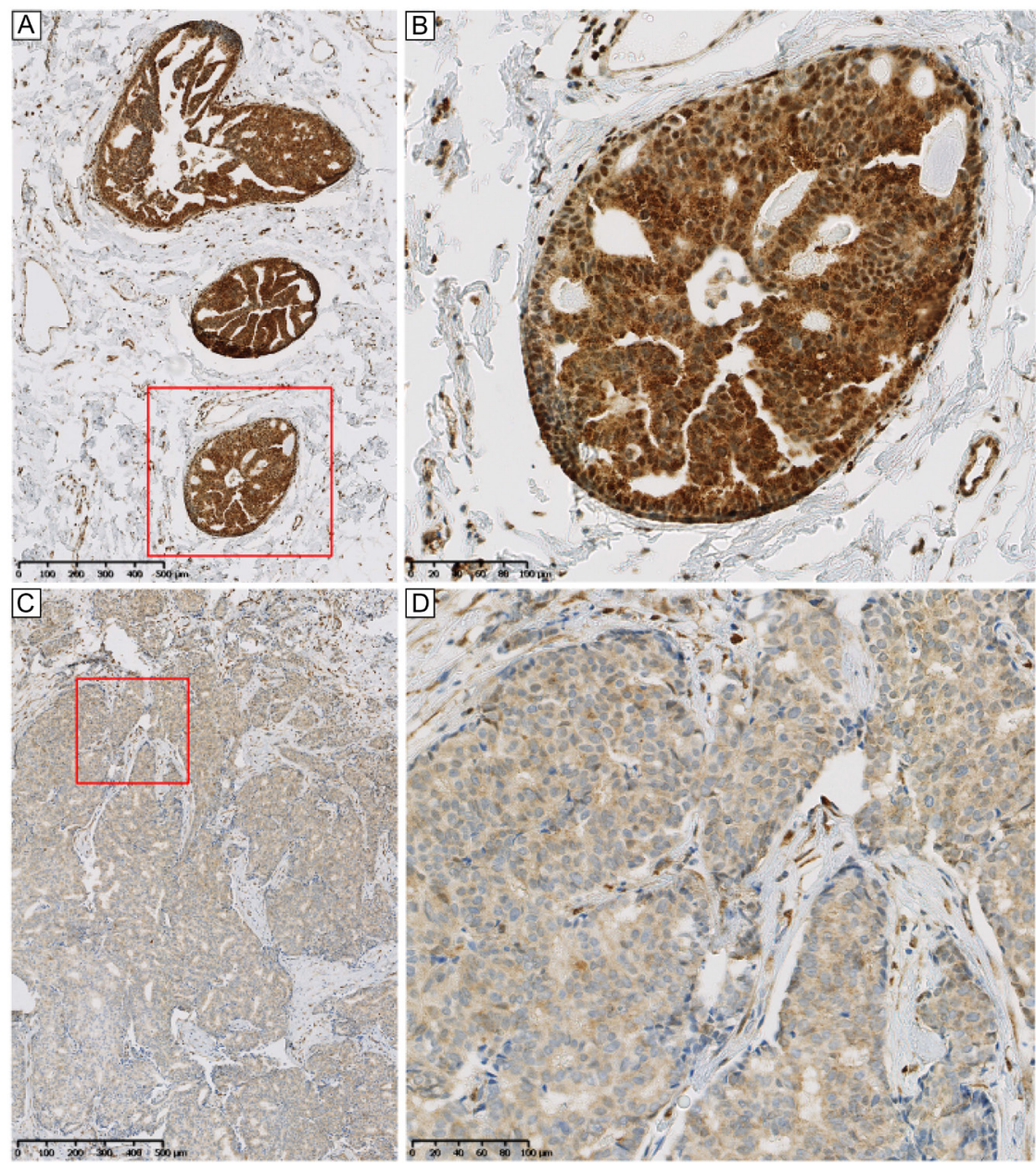

\section{Figure 5}

Illustrative case with a higher IHC expression of PTEN in the DCIS component ( $\mathrm{H}$-score of 300$)$ as compared to the adjacent IBC component (H-score of 115). (A) An overview image of PTEN expression in DCIS at a $5 \times$ magnification, with a red square indicating the enlarged region (B) at $20 \times$ magnification. (C) An overview image of PTEN expression in IBC at a $5 \times$ magnification, with a red square indicating the area enlarged region (D) at 20x magnification.

Therefore, the aim of this study was to have a better understanding of PIK3CA mutations during breast cancer progression. We identified a PIK3CA mutation in $24.7 \%$ of our cohort (18 out of 73 patients) by SNaPshot analysis. The majority of these patients (14 out of 18) had a PIK3CA mutation in both the DCIS and the IBC component. A discordant PIK3CA mutation status was found in four patients by $\mathrm{SNaPshot}$ analyses. This number was reduced to three patients after validation and quantification by dPCR. Our results are in line with previous reports on PIK3CA mutations in DCIS and adjacent IBC, regardless of the different detection methods used in these studies (Li et al. 2010, Miron et al. 2010, Kalinsky et al. 2011, Ang et al. 2014, Sakr et al. 2014). Our data support the presence of PIK3CA mutation heterogeneity in synchronous DCIS and IBC as previously demonstrated (Miron et al. 2010, Ang et al. 2014). Besides, this underlines the early development of PIK3CA mutations during breast cancer carcinogenesis, specifically in ER+ tumors. However, late development of PIK3CA mutations have also been observed (Dupont Jensen et al. 2011, Gonzalez-Angulo et al. 2011, Sueta et al. 2014).
In those cases, a PIK3CA mutation was found in the metastases and not in the primary tumor, suggesting a role in maintenance of breast cancer metastatic lesions. In our cohort, the low frequency of PIK3CA mutations in lymph node metastasis and the absence of a PIK3CA mutations in distant metastasis suggests otherwise, although the number of metastases was too small for defining conclusions.

After pre-screening by SNaPshot analyses, we used dPCR to validate and quantify our results. All mutations identified by the SNaPshot assay were confirmed by dPCR. However, we identified one additional mutation in the IBC stage, which was not detected by the SNaPshot assay. This detection failure by the SNaPshot assay is most likely due to its detection limit of $5-10 \%$ (Fariña Sarasqueta et al. 2011). The PIK3CA VAF in this sample was rather low (6.2\%). We further quantified the PIK3CA mutation frequency in synchronous DCIS and IBC. Our data showed a significantly higher PIK3CA VAF in the DCIS component compared to the adjacent IBC component, which could not be contributed to differences in the (c) 2019 Society for Endocrinology Published by Bioscientifica Ltd. Printed in Great Britain 


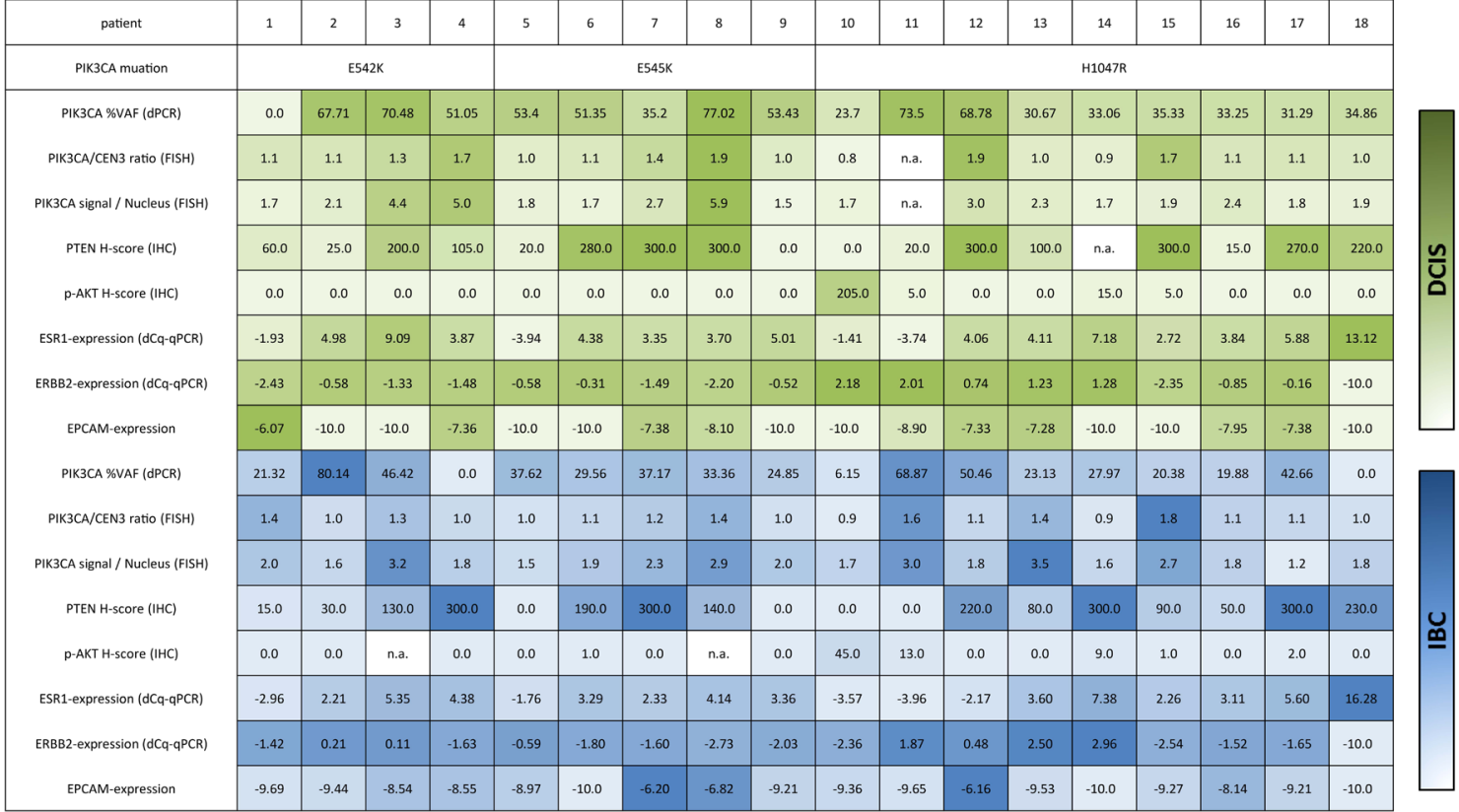

\section{Figure 6}

Heat map of DCIS and IBC results with an overview of performed assays. \%VAF, \% variant allele frequency of PIK3CA; dPCR, digital polymerase chain reaction; FISH, fluorescent in situ hybridization; IHC, immunohistochemistry; qPCR, quantitative PCR. A full colour version of this figure is available at https://doi.org/10.1530/ERC-19-0019.

EPCAM, ESR1 or ERBB2 expression as quantified by RT-qPCR. Previous studies on this topic either focused on the IBC stage only or on high-grade DCIS and synchronous DCIS and IBC (Sakr et al. 2014, Sueta et al. 2014). Sakr and colleagues suggested that PIK3CA mutations in highgrade synchronous DCIS and adjacent IBC are present in a non-modal population of the DCIS component versus a modal population of the IBC cells. These results are rather contradictory to ours, since our data shows the opposite. This outcome could be explained by the use of different detection methods or inclusion criteria. Our results suggest that PIK3CA mutations could be essential in early stages of breast carcinogenesis but might not be necessary for progression from DCIS to IBC. Furthermore, a higher PIK3CA VAF in DCIS compared to IBC suggests subclonal heterogeneity, whereby different clones of malignant cells might have progressed into IBC. However, since PIK3CA mutations are present in both the DCIS stage and the IBC stage in the majority of patients, it could be needed for maintenance of the tumor in the majority of breast cancers.

We detected PIK3CA amplification in the DCIS component of 3 out of 18 patients by FISH. The PIK3CA VAF of two out of these three patients suggests a PIK3CA amplification of the variant PIK3CA allele, since the mutant versus WT allele ratio was up to 3 . Additional investigations in other cohorts have shown important clinical consequences, since acquired PIK3CA amplifications have been associated with resistance to selective phosphoinositide 3-kinase inhibitors in IBC (Huw et al. 2013).

With respect to PTEN and p-AKT scoring, there is no standardized scoring system, nor a standardized cut-off point for positive vs negative. We semi-quantified PTEN and p-AKT expression immunohistochemically using the $\mathrm{H}$-score. In general, PTEN expression was significantly lower in the IBC component compared to the DCIS component. Previous studies reported either a similar PTEN expression or a higher frequency of PTEN loss in the IBC stage compared to the DCIS stage using a dichotomous scoring system (Aktepe et al. 2009, Sakr et al. 2014). The latter study is in line with our finding, suggesting decreased PTEN expression at the IBC stage. When we stratified by PIK3CA mutation status, a lower PTEN expression in IBC compared to DCIS was only observed in WT PIK3CA. In patients with a mutated PIK3CA on the other hand, no significant difference in PTEN expression was observed between these components. In our cohort, we observed co-occurrence of PTEN loss and a PIK3CA mutation in a minority of cases (four IBC samples). Based on these results, we hypothesize that a PIK3CA mutation could contribute to preservation of PTEN expression during progression from DCIS to IBC, since PIK3CA-driven progression does not seem to co-occur with PTEN loss 
in the majority of patients. However, we did not observe significant differences in PTEN expression between the PIK3CA-mutated and WT cases, which might be due to the small cohort. In our cohort, p-AKT was expressed in 15 out of 36 patients. There was no association between PIK3CA mutation status and p-AKT expression: seven patients with p-AKT expression had a PIK3CA mutation, eight patients were WT. There was a strong overlap between p-AKT expression in the DCIS component and the adjacent IBC component. In those patients with p-AKT expression in both components, there was no difference in the level of expression between DCIS and IBC, which is in line with a previous study (Sakr et al. 2014). To the best of our knowledge, this is the first study focusing on the presence and quantity of PIK3CA mutations during the progression from DCIS to IBC, including the potential association with PTEN and p-AKT expression. We are also one of the first to report PIK3CA amplification in breast cancer, specifically in DCIS. Nevertheless, our study also has several limitations. First, the number of mutated cases in our series was rather small for further subgroup analyses. Next, our study is restricted to hotspot changes in the PIK3CA gene only. It is also likely that changes in the PIK3CA gene can further interact with other genes, in and outside the PI3K pathway (Martelotto et al. 2017, Ramirez-Ardila et al. 2017, Wang et al. 2017, Casasent et al. 2018). Besides, we only included patients with a substantial DCIS component to be able to isolate enough DNA to perform our analyses, which could have resulted in a selection bias.

In conclusion, we detected a significantly higher PIK3CA VAF in the DCIS component compared to the adjacent IBC component, which suggests that PIK3CA mutations could specifically be essential in early stages of breast carcinogenesis rather than progression from DCIS to IBC. Moreover, we have shown maintained PTEN expression in DCIS and loss of PTEN expression at the IBC stage, which is associated with a WT PIK3CA. This association suggests that PIK3CA mutations and loss of PTEN expression do not co-occur during DCIS progression to IBC in the majority of patients. In our cohort, there was no association between PIK3CA mutation status and p-AKT expression. Our data might contribute to further unraveling breast carcinogenesis and heterogeneity, which could facilitate patient-specific treatment.

\section{Supplementary data}

This is linked to the online version of the paper at https://doi.org/10.1530/ ERC-19-0019.

\section{Declaration of interest}

The authors declare that there is no conflict of interest that could be perceived as prejudicing the impartiality of the research reported.

\section{Funding}

This study was funded by the Erasmus MC Medical Center and performed at the Erasmus MC Cancer Center at the departments of pathology and medical oncology. The authors thank H J Dubbink, PhD, for critically reading and reviewing our manuscript.

\section{Author contribution statement}

M A G, A M S and C D E conceived and designed experiments; D O E and $C D E$ selected the patients and collected clinical data; M A G, E V E and $\checkmark \mathrm{W}$ E collected and processed the samples; M A G, A M S, C M B, KR R and $\mathrm{H} \mathrm{S} \mathrm{L} \mathrm{conducted} \mathrm{the} \mathrm{experiments;} \mathrm{M} \mathrm{A} \mathrm{G,} \mathrm{A} \mathrm{M} \mathrm{S,} \mathrm{D} \mathrm{I} \mathrm{N,} \mathrm{J} \mathrm{W} \mathrm{M} \mathrm{and}$ $C D E$ consulted and deliberated on the results; $C D E$ secured funding for the project; M A G, A M S and C D E wrote the manuscript. All co-authors reviewed the manuscript.

\section{References}

Abba MC, Gong T, Lu Y, Lee J, Zhong Y, Lacunza E, Butti M, Takata Y, Gaddis S, Shen J, et al. 2015 Molecular portrait of high-grade ductal carcinoma in situ. Cancer Research 75 3980-3990. (https://doi. org/10.1158/0008-5472.CAN-15-0506)

Aktepe F, Kir C, Sahin DA \& Dilek H 2009 Immunohistochemical expression of PTEN and survivin in ductal carcinoma of breast cancer and correlation with apoptotic index. Pathology 41 57-58. (https://doi.org/10.1097/01268031-200941001-00122)

Ang D, Warrick AL, Shilling A, Beadling C, Corless CL \& Troxell ML 2014 Frequent phosphatidylinositol-3-kinase mutations in proliferative breast lesions. Modern Pathology 27 740-750. (https:// doi.org/10.1038/modpathol.2013.197)

Azim HA, Kassem L, Treilleux I, Wang Q, El Enein MA, Anis SE \& Bachelot T 2016 Analysis of PI3K/mTOR pathway biomarkers and their prognostic value in women with hormone receptor-positive, HER2-negative early breast cancer 1. Translational Oncology 9 114-123. (https://doi.org/10.1016/j.tranon.2016.01.001)

Barnes NLP, Ooi JL, Yarnold JR \& Bundred NJ 2012 Ductal carcinoma in situ of the breast how does DCIS develop? BMJ 344 e797. (https:// doi.org/10.1136/bmj.e797)

Beaver JA, Bulakrishna S, Jelovac D, Higgings MJ, Jeter S, Stearns V, Wolff AC, Kessler J, VanDenBerg D, Valda Toro P, et al. 2013 Sensitivity for detecting PIK3CA mutations in early-stage breast cancer with droplet digital PCR. Journal of Clinical Oncology $\mathbf{3 1}$ 11019.

Berns K, Horlings HM, Hennessy BT, Madiredjo M, Hijmans EM, Beelen K, Linn SC, Gonzalez-Angulo AM, Stemke-Hale K, Hauptmann M, et al. 2007 A functional genetic approach identifies the PI3K pathway as a major determinant of trastuzumab resistance in breast cancer. Cancer Cell 12 395-402. (https://doi.org/10.1016/j. ccr.2007.08.030)

Bleyer A \& Welch HG 2012 Effect of three decades of screening mammography on breast-cancer incidence. New England Journal of Medicine 367 1998-2005. (https://doi.org/10.1056/NEJMoa1206809)

Boland GP, Mckeown A, Chan KC, Prasad R, Knox WF \& Bundred NJ 2003 Biological response to hormonal manipulation in oestrogen receptor positive ductal carcinoma in situ of the breast. British (c) 2019 Society for Endocrinology Published by Bioscientifica Ltd. Printed in Great Britain 
Journal of Cancer 89 277-283. (https://doi.org/10.1038/sj. bjc.6601013)

Bose S, Crane A, Hibshoosh H, Mansukhani M, Sandweis L \& Parsons R 2002 Reduced expression of PTEN correlates With breast cancer progression. Human Pathology 33 405-409. (https://doi.org/10.1053/ hupa.2002.124721)

Cancer Genome Atlas Network 2012 Comprehensive molecular portraits of human breast tumours. Nature $\mathbf{4 9 0}$ 61-70. (https://doi. org/10.1038/nature11412)

Casasent AK, Schalck A, Gao R, Sei E, Long A, Pangburn W, Casasent T, Meric-Bernstam F, Edgerton ME \& Navin NE 2018 Multiclonal invasion in breast tumors identified by topographic single cell sequencing. Cell 172 205.e12-217.e12. (https://doi.org/10.1016/j. cell.2017.12.007)

cBioPortal 2015 In cBioPortal for cancer genomics: PIK3CA and PTEN in invasive breast cancer. New York, NY, USA: Memorial Sloan Kettering Cancer Center. (available at: http://www.cbioportal.org/index. do?cancer_study_id=all\&Z_SCORE_THRESHOLD $=2.0 \& R P P A \_S C O R E \_$ THRESHOLD $=2.0 \&$ data_priority $=0 \&$ case_set_id=all\&gene_list=PIK3C A\%2520PTEN\%2520AKT1\&geneset_list=\&tab_index=tab_ visualize\&Action=Submit\&cancer_study_list=brca_tcga\%2Cb)

Chalhoub N \& Baker SJ 2009 PTEN and the PI3-kinase pathway in cancer. Annual Review of Pathology 4 127-150. (https://doi. org/10.1146/annurev.pathol.4.110807.092311)

Cizkova M, Susini A, Vacher S, Cizeron-Clairac G, Andrieu C, Driouch K, Fourme E, Lidereau R \& Bièche I 2012 PIK3CAmutation impact on survival in breast cancer patients and in ER $\alpha$, PR and ERBB2-based subgroups. Breast Cancer Research 14 R28. (https://doi.org/10.1186/ bcr3113)

Cossu-Rocca P, Orrù S, Muroni MR, Sanges F, Sotgiu G, Ena S, Pira G, Murgia L, Manca A, Uras MG, et al. 2015 Analysis of PIK3CA mutations and activation pathways in triple negative breast cancer. PLOS ONE 10 e0141763. (https://doi.org/10.1371/journal. pone.0141763)

Doebar SC, Sieuwerts AM, de Weerd V, Stoop H, Martens JWM \& van Deurzen CHM 2017 Gene expression differences between ductal carcinoma in situ with and without progression to invasive breast cancer. American Journal of Pathology 187 1648-1655. (https://doi. org/10.1016/j.ajpath.2017.03.012)

Dupont Jensen J, Laenkholm AV, Knoop A, Ewertz M, Bandaru R, Liu W, Hackl W, Barrett JC \& Gardner H 2011 PIK3CA mutations may be discordant between primary and corresponding metastatic disease in breast cancer. Clinical Cancer Research 17 667-677. (https://doi. org/10.1158/1078-0432.CCR-10-1133)

Elston CW \& Ellis IO 1991 Pathological prognostic factors in breast cancer. I. The value of histological grade in breast cancer: experience from a large study with long-term follow-up. Histopathology 19 403-410. (https://doi.org/10.1111/j.1365-2559.1991.tb00229.x)

Esteva FJ, Guo H, Zhang S, Santa-Maria C, Stone S, Lanchbury JS, Sahin AA, Hortobagyi GN \& Yu D 2010 PTEN, PIK3CA, p-AKT, and p-p70S6K status association with trastuzumab response and survival in patients with HER2-positive metastatic breast cancer. American Journal of Pathology 177 1647-1656. (https://doi.org/10.2353/ ajpath.2010.090885)

Fariña Sarasqueta A, Moerland E, de Bruyne H, de Graaf H, Vrancken T, van Lijnschoten G \& van den Brule AJC 2011 SNaPshot and StripAssay as valuable alternatives to direct sequencing for KRAS mutation detection in colon cancer routine diagnostics. Journal of Molecular Diagnostics 13 199-205. (https://doi.org/10.1016/j. jmoldx.2010.10.006)

FEDERA 2011 Human Tissue and Medical Research: Code of Conduct for Responsible Use (2011). Rotterdam, Netherlands: Foundation Federation of Dutch Medical Scientific Societies. (available at: https://www.federa.org/sites/default/files/digital_version_first_part_ code_of_conduct_in_uk_2011_12092012.pdf)
Goldhirsch A, Winer EP, Coates AS, Gelber RD, Piccart-Gebhart M, Thürlimann B, Senn HJ \& Panel members 2013 Personalizing the treatment of women with early breast cancer: highlights of the st gallen international expert consensus on the primary therapy of early breast cancer 2013. Annals of Oncology 24 2206-2223. (https:// doi.org/10.1093/annonc/mdt303)

Gonzalez-Angulo AM, Ferrer-Lozano J, Stemke-Hale K, Sahin A, Liu S, Barrera JA, Burgues O, Lluch AM, Chen H, Hortobagyi GN, et al. 2011 PI3K pathway mutations and PTEN levels in primary and metastatic breast cancer. Molecular Cancer Therapeutics 10 1093-1101. (https://doi.org/10.1158/1535-7163.MCT-10-1089)

Gschwantler-Kaulich D, Tan YY, Fuchs EM, Hudelist G, Köstler WJ, Reiner A, Leser C, Salama M, Attems J, Deutschmann C, et al. 2017 PTEN expression as a predictor for the response to trastuzumabbased therapy in Her-2 overexpressing metastatic breast cancer. PLOS ONE 12 e0172911. (https://doi.org/10.1371/journal. pone.0172911)

Guarneri V, Dieci MV, Frassoldati A, Maiorana A, Ficarra G, Bettelli S, Tagliafico E, Bicciato S, Generali DG, Cagossi K, et al. 2015 Prospective biomarker analysis of the randomized Cher-LOB study evaluating the dual anti-HER2 treatment with trastuzumab and lapatinib plus chemotherapy as neoadjuvant therapy for HER2positive breast cancer. Oncologist 20 1001-1010. (https://doi. org/10.1634/theoncologist.2015-0138)

Hernandez L, Wilkerson PM, Lambros MB, Campion-Flora A, Rodrigues DN, Gauthier A, Cabral C, Pawar V, Mackay A, A'Hern R, et al. 2012 Genomic and mutational profiling of ductal carcinomas in situ and matched adjacent invasive breast cancers reveals intratumour genetic heterogeneity and clonal selection. Journal of Pathology 227 42-52. (https://doi.org/10.1002/path.3990)

Hurst CD, Zuiverloon TC, Hafner C, Zwarthoff EC \& Knowles MA 2009 A SNaPshot assay for the rapid and simple detection of four common hotspot codon mutations in the PIK3CA gene. BMC Research Notes 2 66. (https://doi.org/10.1186/1756-0500-2-66)

Huw LY, O'Brien C, Pandita A, Mohan S, Spoerke JM, Lu S, Wang Y, Hampton GM, Wilson TR \& Lackner MR 2013 Acquired PIK3CA amplification causes resistance to selective phosphoinositide 3-kinase inhibitors in breast cancer. Oncogenesis 2 e83. (https://doi. org/10.1038/oncsis.2013.46)

Iqbal J, Thike AA, Cheok PY, Tse GM-K \& Tan PH 2012 Insulin growth factor receptor-1 expression and loss of PTEN protein predict early recurrence in triple-negative breast cancer. Histopathology $\mathbf{6 1}$ 652-659. (https://doi.org/10.1111/j.1365-2559.2012.04255.x)

Kalinsky K, Heguy A, Bhanot UK, Patil S \& Moynahan ME 2011 PIK3CA mutations rarely demonstrate genotypic intratumoral heterogeneity and are selected for in breast cancer progression. Breast Cancer Research and Treatment 129 635-643. (https://doi.org/10.1007/ s10549-011-1601-4)

Kalinsky K, Jacks LM, Heguy A, Patil S, Drobnjak M, Bhanot UK, Hedvat CV, Traina TA, Solit D, Gerald W, et al. 2009 Human cancer biology PIK3CA mutation associates with improved outcome in breast cancer. Clinical Cancer Research 15 5049-5059. (https://doi. org/10.1158/1078-0432.CCR-09-0632)

Kim JH, Lee JS, Kim EJ, Park KH, Kim KH, Yi SY, Kim HS, Cho YJ, Shin KH, Ahn JB, et al. 2016 Prognostic implications of PIK3CA amplification in curatively resected liposarcoma. Oncotarget $\mathbf{7}$ 24549-24558. (https://doi.org/10.18632/oncotarget.8240)

Knudsen ES, Pajak TF, Qeenan M, McClendon AK, Armon BD, Schwartz GF \& Witkiewicz AK 2012 Retinoblastoma and phosphate and tensin homolog tumor suppressors: impact on ductal carcinoma in situ progression. Journal of the National Cancer Institute $\mathbf{1 0 4}$ 1825-1836. (https://doi.org/10.1093/jnci/djs446)

Lee JS, Kim HS, Kim YB, Lee MC, Park CS \& Min KW 2004 Reduced PTEN expression is associated with poor outcome and angiogenesis in invasive ductal carcinoma of the breast. Applied (c) 2019 Society for Endocrinology Published by Bioscientifica Ltd. Printed in Great Britain 
Immunohistochemistry and Molecular Morphology 12 205-210. (https:// doi.org/10.1097/00129039-200409000-00004)

Li H, Zhu R, Wang L, Zhu T, Li Q, Chen Q, Wang H \& Zhu H 2010 PIK3CA mutations mostly begin to develop in ductal carcinoma of the breast. Experimental and Molecular Pathology 88 150-155. (https:// doi.org/10.1016/j.yexmp.2009.09.016)

Li S, Shen Y, Wang M, Yang J, Lv M, Li P, Chen Z \& Yang J 2017 Loss of PTEN expression in breast cancer: association with clinicopathological characteristics and prognosis. Oncotarget 8 32043-32054. (https://doi.org/10.18632/oncotarget.16761)

Loi S, Haibe-Kains B, Majjaj S, Lallemand F, Durbecq V, Larsimont D, Gonzalez-Angulo AM, Pusztai L, Fraser Symmans WF, Bardelli A, et al. 2010 PIK3CA mutations associated with gene signature of low mTORC1 signaling and better outcomes in estrogen receptorpositive breast cancer. PNAS 107 10208-10213. (https://doi. org/10.1073/pnas.0907011107)

Martelotto LG, Baslan T, Kendall J, Geyer FC, Burke KA, Spraggon L, Piscuoglio S, Chadalavada K, Nanjangud G, Ng CKY, et al. 2017 Whole-genome single-cell copy number profiling from formalinfixed paraffin-embedded samples. Nature Medicine 23 376-385. (https://doi.org/10.1038/nm.4279)

Miron A, Varadi M, Carrasco D, Li H, Luongo L, Kim HJ, Park SY, Cho EY, Lewis G, Kehoe S, et al. 2010 PIK3CA mutations in in situ and invasive breast carcinomas. Cancer Research 70 5674-5678. (https://doi.org/10.1158/0008-5472.CAN-08-2660)

Mukohara T 2015 PI3K mutations in breast cancer: prognostic and therapeutic implications. Breast Cancer (Dove Medical Press) 7 111-123. (https://doi.org/10.2147/BCTT.S60696)

NABON 2017 Factsheet Indicatoren NABON Breast Cancer Audit (NBCA). Leiden, Netherlands: Dutch Institute for Clinical Auditing. (available at: https://www.zorginzicht.nl/bibliotheek/Borstkanker/ RegisterMeetinstrumentenDocumenten/Indicatorgids $\% 20$ Mammacarcinoom\%20(NBCA)\%20verslagjaar\%202017.pdf)

Nagata Y, Lan K-H, Zhou X, Tan M, Esteva FJ, Sahin AA, Klos KS, Li P, Monia BP, Nguyen NT, et al. 2004 PTEN activation contributes to tumor inhibition by trastuzumab, and loss of PTEN predicts trastuzumab resistance in patients. Cancer Cell 6 117-127. (https:// doi.org/10.1016/j.ccr.2004.06.022)

Pang B, Cheng S, Sun SP, An C, Liu ZY, Feng X \& Liu GJ 2014 Prognostic role of PIK3CA mutations and their association with hormone receptor expression in breast cancer: a meta-analysis. Scientific Reports 4 6255. (https://doi.org/10.1038/srep06255)

Perou CM, Sùrlie T, Eisen MB, Rijn M, Van De Jeffrey SS, Rees CA, Pollack JR, Ross DT, Johnsen H, Akslen LA, et al. 2000 Molecular portraits breast cancer. Nature 533 747-752.

Ramirez-Ardila D, Timmermans AM, Helmijr JA, Martens JWM, Berns EMJJ \& Jansen MPHM 2017 Increased MAPK1/3 phosphorylation in luminal breast cancer related with PIK3CA hotspot mutations and prognosis. Translational Oncology 10 854-866. (https://doi.org/10.1016/j.tranon.2017.08.002)

Saal LH, Holm K, Maurer M, Memeo L, Su T, Wang X, Yu JS, Malmström PO, Mansukhani M, Enoksson J, et al. 2005 PIK3CA mutations correlate with hormone receptors, node metastasis, and ERBB2, and are mutually exclusive with PTEN loss in human breast carcinoma. Cancer Research 65 2554-2559. (https://doi. org/10.1158/0008-5472-CAN-04-3913)

Sakr RA, Barbashina V, Morrogh M, Chandarlapaty S, Andrade VP, Arroyo CD, Olvera N \& King TA 2010 Protocol for PTEN expression by immunohistochemistry in formalin-fixed paraffin-embedded human breast carcinoma. Applied Immunohistochemistry and Molecular
Morphology 18 371-374. (https://doi.org/10.1097/ PAI.0b013e3181d50bd5)

Sakr RA, Weigelt B, Chandarlapaty S, Andrade VP, Guerini-Rocco E, Giri D, Ng CKY, Cowell CF, Rosen N, Reis-Filho JS, et al. 2014 Human cancer biology PI3K pathway activation in high-grade ductal carcinoma in situ - implications for progression to invasive breast carcinoma. Clinical Cancer Research 20 2326-2337. (https://doi. org/10.1158/1078-0432.CCR-13-2267)

Shi J, Yao D, Liu W, Wang N, Lv H, Zhang G, Ji M, Xu L, He N, Shi B, et al. 2012 Highly frequent PIK3CA amplification is associated with poor prognosis in gastric cancer. BMC Cancer 12 50. (https://doi. org/10.1186/1471-2407-12-50)

Sieuwerts AM, Schrijver WAME, Dalm SU, de Weerd V, Moelans CB, ter Hoeve N, van Diest PJ, Martens JWM \& van Deurzen CHM 2017 Progressive APOBEC3B mRNA expression in distant breast cancer metastases. PLOS ONE 12 e0171343. (https://doi.org/10.1371/journal. pone.0171343)

Song CH, Park SY, Eom KY, Kim JH, Kim SW, Kim JS \& Kim IA 2010 Potential prognostic value of heat-shock protein 90 in the presence of phosphatidylinositol-3-kinase overexpression or loss of PTEN, in invasive breast cancers. Breast Cancer Research 12 R20. (https://doi. org/10.1186/bcr2557)

Sueta A, Yamamoto Y, Yamamoto-Ibusuki M, Hayashi M, Takeshita T, Yamamoto S \& Iwase H 2014 An integrative analysis of PIK3CA mutation, PTEN, and INPP4B expression in terms of trastuzumab efficacy in HER2-positive breast cancer. PLOS ONE 9 e116054. (https://doi.org/10.1371/journal.pone.0116054)

Troxell ML 2012 PIK3CA/AKT1 mutations in breast carcinoma: a comprehensive review of experimental and clinical studies. Journal of Clinical and Experimental Pathology $\mathbf{S 1}$ 002. (https://doi. org/10.4172/2161-0681.S1-002)

Vivanco I \& Sawyers CL 2002 The phosphatidylinositol 3-kinase-AKT pathway in human cancer. Nature Reviews Cancer 2 489-501. (https:// doi.org/10.1038/nrc839)

Vogt PK, Kang S, Elsliger MA \& Gymnopoulos M 2007 Cancer-specific mutations in phosphatidylinositol 3-kinase. Trends in Biochemical Sciences 32 342-349. (https://doi.org/10.1016/j.tibs.2007.05.005)

Wang J, Zhu X, Xu X, Guo L, Shen G, Liu X, Chang C, Wang B, Yang H \& Wang M 2017 PIK3CA mutations and downstream effector p-mTOR expression: implication for prognostic factors and therapeutic targets in triple negative breast cancer. International Journal of Clinical and Experimental Pathology 10 7682-7691.

Wang LL, Hao S, Zhang S, Guo LJ, Hu CY, Zhang G, Gao B, Zhao JJ, Jiang Y, Tian WG, et al. 2017 PTEN/PI3K/AKT protein expression is related to clinicopathological features and prognosis in breast cancer with axillary lymph node metastases. Human Pathology 61 49-57. (https://doi.org/10.1016/j.humpath.2016.07.040)

Wolff AC, Hammond MEH, Hicks DG, Dowsett M, McShane LM, Allison KH, Allred DC, Bartett JMS, Bilous M, Fitzgibbons P, et al. 2014 Recommendations for human epidermal growth factor receptor 2 testing in breast cancer: American Society of Clinical Oncology/ College of American Pathologists clinical practice guideline update. Archives of Pathology and Laboratory Medicine 138 241-256. (https:// doi.org/10.5858/arpa.2013-0953-SA)

Zardavas D, Te Marvelde L, Milne RL, Fumagalli D, Fountzilas G, Kotoula V, Razis E, Papaxoinis G, Joensuu H, Moynahan ME, et al. 2018 Tumor PIK3CA genotype and prognosis in early-stage breast cancer: a pooled analysis of individual patient data. Journal of Clinical Oncology 36 981-990. (https://doi.org/10.1200/ JCO.2017.74.8301)

Received in final form 1 March 2019

Accepted 6 March 2019

Accepted Preprint published online 6 March 2019 https://erc bioscientifica com https://doi.org/10.1530/ERC-19-0019 (c) 2019 Society for Endocrinology Published by Bioscientifica Ltd. Printed in Great Britain 